\title{
Akt inhibitor shows anticancer and radiosensitizing effects in malignant glioma cells by inducing autophagy
}

\author{
KEISHI FUJIWARA ${ }^{1}$, EIJI IWADO ${ }^{1}$, GORDON B. MILLS ${ }^{2}$, \\ RAYMOND SAWAYA $^{1,3}$, SEIJI KONDO ${ }^{1,3,4}$ and YASUKO KONDO ${ }^{1}$ \\ Departments of ${ }^{1}$ Neurosurgery and ${ }^{2}$ Molecular Therapeutics, The University of Texas \\ M.D. Anderson Cancer Center; ${ }^{3}$ Department of Neurosurgery, The Baylor College of Medicine; \\ ${ }^{4}$ The University of Texas Graduate School of Biomedical Sciences at Houston, Houston, TX, USA
}

Received March 26, 2007; Accepted May 17, 2007

\begin{abstract}
Autophagy, or programmed cell death type II, is one of the responses of cancer cells to various therapies, including ionizing radiation. Recently, we have shown that radiation induces autophagy, but not apoptosis, in various malignant glioma cell lines. Autophagy is mainly regulated by the mammalian target of rapamycin (mTOR) pathway. The Akt/ mTOR pathway also mediates oncogenesis and radioresistance. Thus, we hypothesized that inhibiting this pathway has both an anticancer and radiosensitizing effect by activating autophagy. The purpose of our study was therefore to determine whether and by which mechanisms an Akt inhibitor, 1L-6hydroxymethyl-chiro-inositol 2(R)-2-O-methyl-3-Ooctadecylcarbonate, had anticancer and radiosensitizing effects on malignant glioma U87-MG and radioresistant U87-MG cells with a consistitutively active form of epidermal growth factor receptor (U87-MG $\triangle \mathrm{EGFR}$ ). Treatment with the Akt inhibitor successfully inhibited Akt activity and reduced cell viability in both cell lines. In terms of the mechanism, the Akt inhibitor decreased phosphorylated p70S6 kinase, a downstream target of Akt, and induced autophagy, but not apoptosis. Furthermore, the Akt inhibitor radiosensitized both U87-MG and U87-MG $\triangle E G F R$ cells by enhancing autophagy.
\end{abstract}

Correspondence to: Dr Seiji Kondo or Dr Yasuko Kondo, Department of Neurosurgery, Unit BSRB1004, The University of Texas M.D. Anderson Cancer Center, 1515 Holcombe Boulevard, Houston, TX 77030, USA

E-mail: seikondo@mdanderson.org; yaskondo@mdanderson.org

Abbreviations: TOR, target of rapamycin; mTOR, mammalian TOR; PI3K, phosphatidylinositol 3 kinase; p70S6K, p70S6 kinase; EGFR, epidermal growth factor receptor; GFP, green fluorescent protein; LC3, microtubule-associated protein 1 light chain 3; HA, hemagglutinin; PTEN, phosphatase and tensin homolog deleted on chromosome ten; PDGF, platelet-derived growth factor

Key words: Akt inhibitor, radiosensitization, autophagy, malignant glioma
Specific inhibition of Akt using the dominant-negative Akt plasmid also resulted in enhanced radiation-induced autophagy. In conclusion, an Akt inhibitor showed anticancer and radiosensitizing effect on U87-MG and U87-MG $\triangle$ EGFR cells by inducing autophagy. Thus, Akt inhibitors may represent a promising new therapy as a single treatment or used in combination with radiation for malignant gliomas, including radioresistant ones that express $\triangle \mathrm{EGFR}$.

\section{Introduction}

Autophagy is a newly identified response of cancer cells to various anticancer therapies such as chemotherapy and radiotherapy (1-3). Anticancer therapies that induce autophagy in cancer cells include the anti-estrogen receptor agent tamoxifen in breast cancer cells (4), the alkylating agent temozolomide in malignant glioma cells (5), and ionizing radiation in breast cancer, colon cancer, prostate cancer, and malignant glioma cells (6-9). Autophagic cell death is referred to as programmed cell death type II; programmed cell death type I is apoptosis $(4,10)$. These two types of cell death are usually distinctive. Autophagic cell death is characterized by the formation of numerous autophagic vacuoles that contain cytoplasmic proteins and subcellular organelles such as mitochondria; apoptosis is characterized by the condensation and fragmentation of the nucleus.

Autophagy is basically a protein degradation system and its regulatory pathways have been extensively studied in the setting of starvation-induced autophagy. It is widely known that target of rapamycin (TOR) or mammalian TOR (mTOR) plays a central role in regulating autophagy in from yeast to mammalian cells $(11,12)$. mTOR inhibits autophagy predominantly by activating a downstream molecule, p70S6 kinase (p70S6K). In line with this mechanism, rapamycin has been observed to induce autophagy by inhibiting mTOR (13). On the other hand, mTOR is a downstream molecule of the class I phosphatidylinositol 3 kinase (PI3K)/Akt pathway. Thus, PTEN, an inhibitor of PI3K, induces autophagy and Akt inhibits it (14).

Dysregulation of the signaling of the epidermal growth factor receptor (EGFR) is common in various cancers, including malignant gliomas. The most frequent mutation of 
the EGFR gene observed in malignant gliomas is a deletion of exons 2-7, which results in a constitutively active form of EGFR ( $\triangle$ EGFR) $(15,16)$. The downstream PI3K/Akt/mTOR pathway is activated in these tumors, thereby increasing cancer cell survival, proliferation, and angiogenesis. Further, multiple studies have shown that $\triangle E G F R$ confers radioresistance $(17,18)$. These findings strongly support inhibition of the PI3K/ Akt/mTOR pathway as a promising strategy for treatment of malignant gliomas both as a single agent and a radiosensitizer $(19,20)$. LY294002 and rapamycin are two inhibitors of this pathway that have frequently been used in research: LY294002 inhibits PI3K, and rapamycin inhibits mTOR. However, these compounds have some drawbacks from a therapeutic standpoint, in that LY294002 inhibits pathways other than Akt/ mTOR, thereby inducing adverse side-effects, and rapamycin inhibits only a part of mTOR activity, thus achieving only a modest anticancer effect. Therefore, the effects of both directly and efficiently inhibiting the Akt/mTOR pathway need to be explored.

1L-6-Hydroxymethyl-chiro-inositol 2(R)-2-O-methyl-3O-octadecylcarbonate (Akt inhibitor) is a new Akt inhibitor that was chosen from among several phosphatidylinositol analogues as the lead compound that selectively inhibits Akt activity (21). In a previous study, Akt inhibitor was found to sensitize different leukemia cell lines to chemotherapeutic drugs and radiation and also to enhance the apoptosis induced by these therapies (22). Since malignant glioma cells are generally very resistant to apoptosis induced by various anticancer therapies, including radiation, this agent is very attractive as a potential means of improving the therapeutic ratio in this now deadly cancer. In the present study, we therefore examined the antitumor and radiosensitizing effects of Akt inhibitor on cell growth and cell death in human malignant glioma U87-MG and U87-MG $\triangle E G F R$ cells. We found that this inhibitor induced autophagic cell death in both cell types and that it radiosensitized these cells by enhancing autophagy.

\section{Materials and methods}

Cell lines. Human malignant glioma U87-MG cells were purchased from the American Type Culture Collection (Manassas, VA), and U87-MG $\triangle$ EGFR cells were kindly provided by Dr W.K. Cavenee (16). Cells were cultured in Dulbecco's modified Eagle's medium (DMEM) supplemented with $10 \%$ fetal bovine serum (FBS) (Invitrogen, Carlsbad, CA), $4 \mathrm{mM}$ glutamine, $100 \mathrm{U} / \mathrm{ml}$ penicillin, and $100 \mu \mathrm{g} / \mathrm{ml}$ streptomycin at $37^{\circ} \mathrm{C}$ in a $5 \% \mathrm{CO}_{2}$ incubator. For the purpose of serum deprivation, cells were incubated in DMEM with $0.5 \% \mathrm{FBS}$ for $24 \mathrm{~h}$ before assays or treatments.

Reagents. 1L-6-Hydroxymethyl-chiro-inositol 2(R)-2-Omethyl-3-O-octadecylcarbonate (Akt inhibitor) (Calbiochem, San Diego, CA) $(21,22)$ was prepared in DMSO and stored at $-20^{\circ} \mathrm{C}$. Cells were serum-deprived for $24 \mathrm{~h}$ prior to treatment with Akt. Acridine orange was purchased from Polysciences (Warrington, PA).

Ionizing radiation, viable cell counting, and clonogenic survival assay. Radiation treatment was administered using a cesium 137 irradiator (model E-0103; U.S. Nuclear Corp., Burbank, CA) at a dose rate of $3.216 \mathrm{~Gy} / \mathrm{min}$. For the viable cell counts, cells exposed to various doses of radiation were seeded in 96-well plates at a density of 10,000 cells/well. One to three days after irradiation, cells were detached by trypsinization, and the number of viable cells was counted by a trypan blue dye exclusion assay. For the clonogenic survival assay, cells were pretreated with or without $40 \mu \mathrm{M} \mathrm{Akt}$ inhibitor for $24 \mathrm{~h}$ under serum-deprived conditions and then irradiated with various doses. Irradiated cells were seeded in 6-well plates in DMEM with 10\% FBS, incubated for 14 days, and then fixed and stained with $0.5 \%$ crystal violet (Sigma, St. Louis, MO)/methanol. Colonies that contained 50 or more cells were counted as described previously (9). The percent plating efficiency and fractions of surviving cells following given treatments were calculated on the basis of the survival of nonirradiated cells not treated with any combination of treatment.

Immunoblotting. Cells were serum-deprived for $24 \mathrm{~h}$ and subjected to immunoblotting to exclude the possible effect of growth factors in the serum. The cells were lysed using lysis buffer (10 mM Tris-HCl, pH 7.8, 1\% NP40, $150 \mathrm{mM} \mathrm{NaCl,}$ $1 \mathrm{mM}$ EDTA) for $10 \mathrm{~min}$ and centrifuged at 15,000 $\mathrm{x} \mathrm{g}$ for $10 \mathrm{~min}$ at $4^{\circ} \mathrm{C}$. Proteins ( $40 \mu \mathrm{g}$ per sample) from the supernatant were separated by $10 \%$ SDS-PAGE and transferred to Hybond-P membranes (Amersham, Piscataway, NJ), as described previously (9). Membranes were incubated with primary antibodies and then with corresponding horseradish peroxidase-conjugated secondary antibodies (Amersham). The bound antibody complex was detected by an enhanced chemiluminescence (ECL) or ECL-plus reagent (Amersham). The antibodies used were rabbit anti-phospho-Akt $\left(\mathrm{Ser}^{473}\right)$, total Akt, phospho-p70S6K ( $\mathrm{Thr}^{389}$ ), and total p70S6K (Cell Signaling, Beverly, MA). For loading controls, membranes were reprobed with total Akt or total p70S6K antibody.

Akt activity assay. For the Akt activity assay, cells were washed with phosphate-buffered saline (PBS) and lysed in lysis buffer (1\% TritonX-100, $50 \mathrm{mM}$ HEPES pH 7.4, $150 \mathrm{mM}$ $\mathrm{NaCl}, 1 \mathrm{mM}$ EGTA, $10 \mathrm{mM}$ sodium pyrophosphate, $1.5 \mathrm{mM}$ $\mathrm{MgCl}_{2}, 100 \mathrm{mM}$ sodium fluoride, $10 \%$ glycerol, $1 \mathrm{mM}$ $\mathrm{Na}_{3} \mathrm{VO}_{4}, 1 \mathrm{mM}$ PMSF, and $10 \mu \mathrm{g} / \mathrm{ml}$ aprotinin). After microcentrifugation for $10 \mathrm{~min}$ at $4^{\circ} \mathrm{C}$, supernatants were stored at $-80^{\circ} \mathrm{C}$ until use. The protein concentration was adjusted to $1 \mathrm{mg} / \mathrm{ml}$ through the addition of lysis buffer. Cell extracts $(200 \mu \mathrm{l})$ were incubated for $2 \mathrm{~h}$ with immobilized Akt (1G1) mouse monoclonal antibody (Cell Signaling). After the cells were extensively washed, the kinase reaction was performed in the presence of $200 \mu \mathrm{M}$ cold ATP and GSK-3 substrate. Then, samples were resolved in 10\% SDS-PAGE and the amount of phosphorylated GSK-3 was measured by immunoblotting using the phospho-GSK-3 $\alpha / \beta$ (Ser21/9) antibody (Cell Signaling).

WST-1 cell viability assay. For the cell viability assay, cells were seeded at equal numbers in 96-well plates and incubated overnight. After serum deprivation for $24 \mathrm{~h}$, cells were treated with various concentrations of the Akt inhibitor and incubated for 3 days with 10\% FBS in DMEM. Cell viability 
A

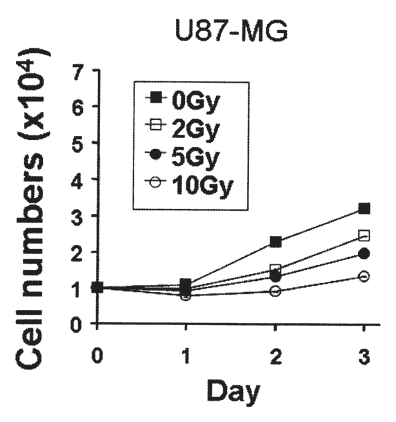

U87-MGAEGFR

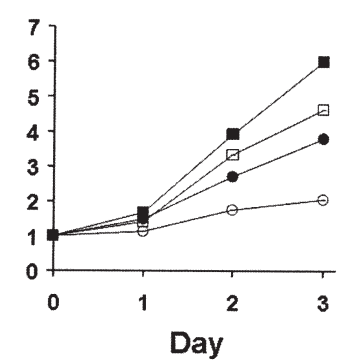

B

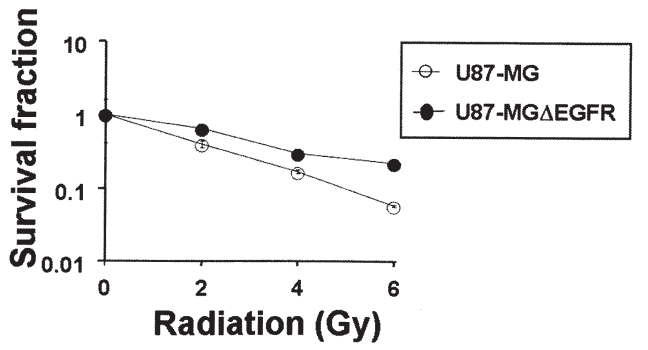

C

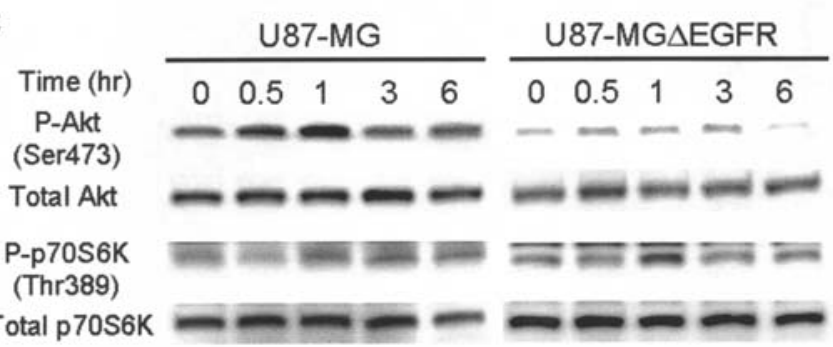

Figure 1. Radiation effect on U87-MG and U87-MG $\triangle E$ EFR cells. A, radiation effect on cell viability. Cells were irradiated at various doses and plated in 96-well plates. After 1-3 days, cells were trypsinized, and viable cells were counted using the trypan blue cell exclusion assay. Values are the mean $\pm \mathrm{SD}$ of triplicate experiments. $\mathrm{B}$, radiation effect on clonogenic survival. Cells were irradiated at various doses and plated at different cell numbers in 6-well plates. After a 14-day incubation, cells were stained with crystal violet, and colonies containing $>50$ cells were counted. Values are the mean \pm SD of triplicate experiments. $\mathrm{C}$, immunoblot analysis of the Akt/p70S6K pathway after irradiation. Cells were serum-deprived for $24 \mathrm{~h}$, irradiated at $10 \mathrm{~Gy}$, and subjected to immunoblotting at the indicated time points. The data shown are representative of three independent experiments.

was determined with the cell proliferation reagent WST-1 colorimetric assay (Roche Applied Science, Indianapolis, IN), according to the manufacturer's instructions.

Cell cycle analysis. Both detached and attached cells were collected, fixed with ice-cold $70 \%$ ethanol, and stained with propidium iodide with the cellular DNA flow cytometric analysis reagent set (Boehringer Mannheim, Indianapolis, IN). The cell cycle profile was analyzed for DNA content using a FACScan (Becton Dickinson, San Jose, CA). Data were analyzed with CellQuest software (Becton Dickinson).

Electron microscopy. Cells were grown on glass coverslips, treated or not treated with Akt inhibitor, and incubated for 3 days. Then, the cells were fixed with a solution containing $3 \%$ glutaraldehyde plus $2 \%$ paraformaldehyde in $0.1 \mathrm{M}$ cacodylate buffer, $\mathrm{pH} 7.3$ for $1 \mathrm{~h}$. Next, the samples were postfixed in $1 \% \mathrm{OsO}_{4}$ in the same buffer for $1 \mathrm{~h}$ and then
A

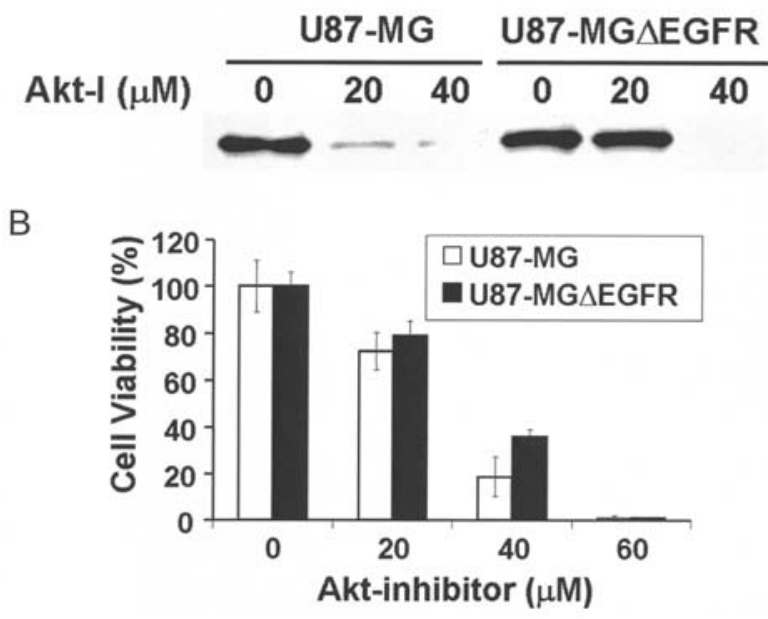

Figure 2. Akt inhibitor inhibits Akt activity and cell viability effectively in U87-MG and U87-MG $\triangle$ EGFR cells. A, Akt activity after treatment with Akt inhibitor. Cells were serum-deprived for $24 \mathrm{~h}$, incubated with Akt inhibitor for $24 \mathrm{~h}$, and cell lysates were isolated. Akt was immunoprecipitated, and Akt activity was assayed. The data shown are representative of two independent experiments. B, cell viability after treatment with Akt inhibitor. Cells were serum-deprived for $24 \mathrm{~h}$, incubated with the Akt inhibitor in DMEM with $10 \%$ FBS for $72 \mathrm{~h}$, and cell viability determined using the WST1 assay. Values are the mean \pm SD of triplicate experiments.

examined under an electron microscope, as described previously (5). Ultra-thin sections were cut and viewed with a JEM 1010 transmission electron microscope (JEOL, Peabody, MA) at an accelerating voltage of $80 \mathrm{kV}$. Digital images were obtained by an AMT imaging system (Advanced Microscopy Techniques Corp., Danvers, MA).

Supravital cell staining with acridine orange. Acidic vesicular organelles were assessed by acridine orange staining, as described previously $(6,9)$. Acridine orange fluoresces green when it binds to DNA and fluoresces a bright red in acidic vesicles, thus green intensity indicates DNA content and red intensity represents acidic vesicles. For quantification of the staining, cells were stained with acridine orange at a final concentration of $1 \mu \mathrm{g} / \mathrm{ml}$ for $15 \mathrm{~min}$ and then removed from the plate by trypsinization. Cells were analyzed by flow cytometry using a FACScan/CellQuest software system.

cDNA plasmid transfection. The green fluorescent protein (GFP) and microtubule-associated protein 1 light chain 3 (LC3) fusion vector (GFP-LC3) was kindly provided by Dr N. Mizushima (Tokyo Metropolitan Institute of Medical Science, Tokyo, Japan) (23). Cells, which were cultured on the chamber slide dish, were transfected with $1 \mu \mathrm{g}$ of GFP-LC3 expression vector. FuGENE6 Transfection Reagent (Roche) was used as described previously (9). At $24 \mathrm{~h}$ after transfection, cells were given specific treatments. At $72 \mathrm{~h}$ after transfection, cells were fixed with $1 \%$ paraformaldehyde in PBS and examined under a fluorescence microscope. The proportion of the cells with GFP-LC3 dots was assessed in 100 GFPpositive cells in three different fields. A hemagglutinin (HA)tagged myristoylated (Myr) Akt1 expression vector and an 

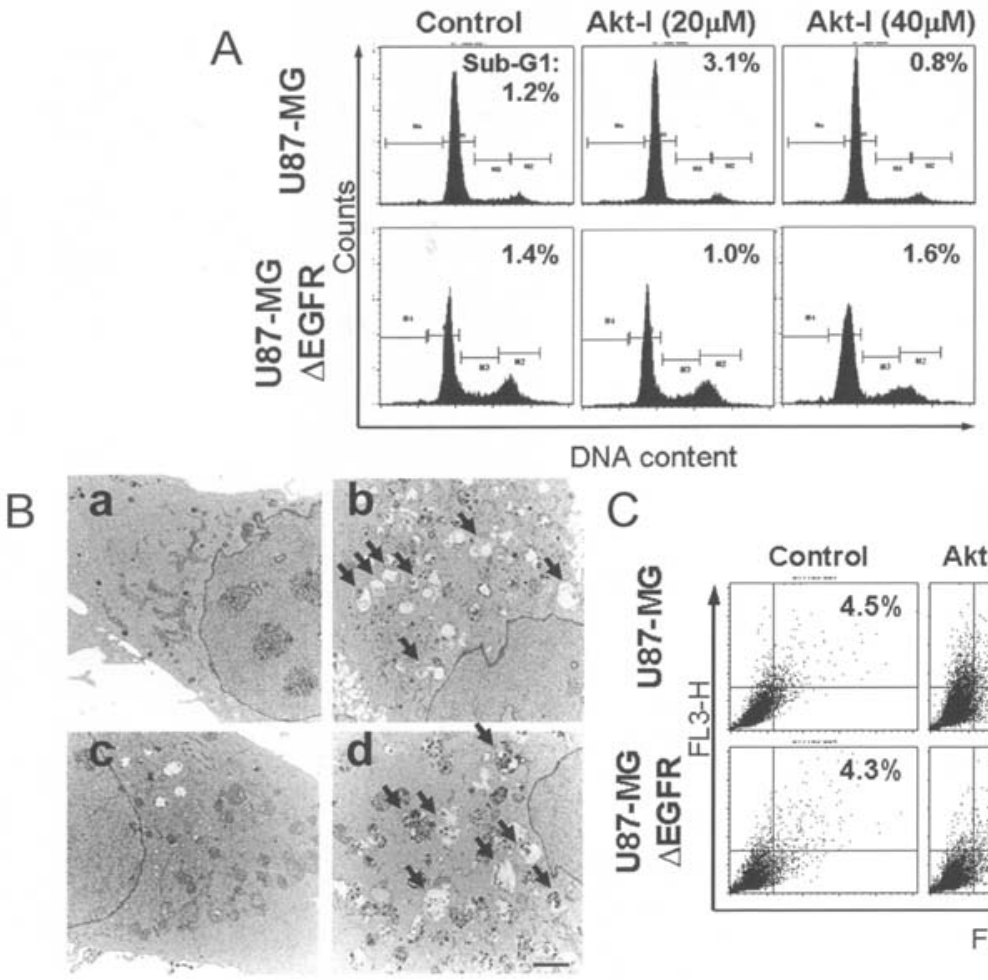

DNA content

D

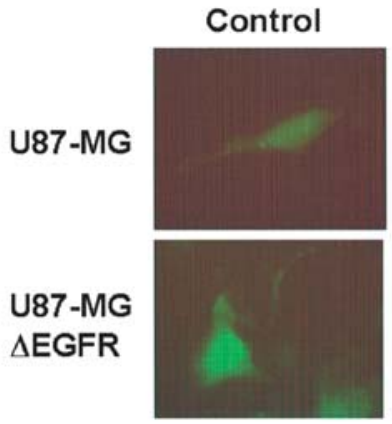

Akt inhibitor

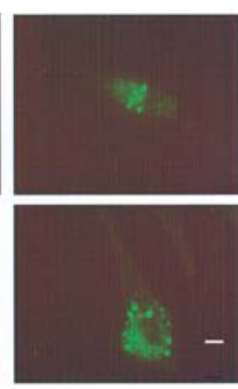

C

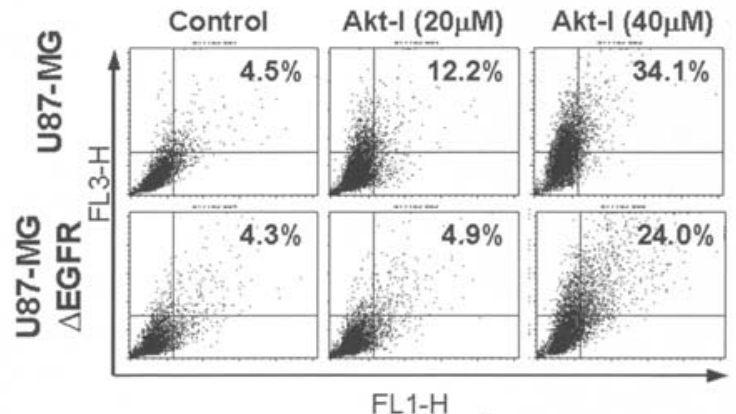

E

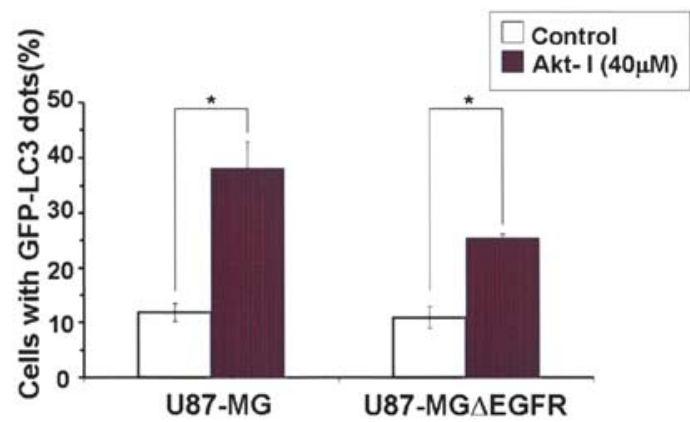

Figure 3. Akt inhibitor induces autophagy, but not apoptosis, in U87-MG and U87-MG $\triangle$ EGFR cells. A, cell cycle analysis. Percentage of the sub-G1 population is indicated. The data shown are representative of three independent experiments. B, representative electron photomicrographs of the cells after incubation with 0 (a and c) and $40 \mu \mathrm{M}$ (b and d) Akt inhibitor for $72 \mathrm{~h}$. Arrows indicate autophagic vacuoles. a and b, U87-MG cells; c and d, U87MG $\triangle E G F R$ cells; scale bar, $2 \mu \mathrm{m}$. C, acridine orange staining assayed with flow cytometry. Percentage of positive-stained cells with red fluorescence is indicated. FL1-H indicates intensity of green fluorescence that is endogenous in the nucleus. FL3-H indicates intensity of red fluorescence. The data shown are representative of three independent experiments. D, representative photomicrographs of the cells transfected with the GFP-LC3 plasmid. Scale bar, $10 \mu \mathrm{m}$. $\mathrm{E}$, quantification of the cells with characteristic GFP-LC3 dots. The proportion of the cells with GFP-LC3 dots was assessed in 100 GFP-positive cells in three different fields. Values are the mean $\pm \mathrm{SD}$ of triplicate experiments. ${ }^{*} \mathrm{P}<0.001$.

HA-tagged dominant-negative (DN) Akt expression vector were also used, as described previously (13). Cells were co-transfected with Myr- or DN-Akt and GFP-LC3 vectors before treatment.

Statistical analysis. The data were expressed as the mean \pm standard deviation (SD). A Student's t-test (two-tailed) was performed to determine statistical significance. The criterion for statistical significance was $\mathrm{P}<0.05$.

\section{Results}

Ionizing radiation induces Akt activity in U87-MG and U87$M G \triangle E G F R$ cells. $\triangle \mathrm{EGFR}$ has been reported to confer radioresistance to cancer cells $(17,18)$. To compare radiation effect on U87-MG and U87-MG $\triangle E G F R$ cells, we determined both the short- and long-term effect of radiation on these cell lines. Radiation inhibited cell proliferation in a dose-dependent manner for 3 days, but the number of viable cells of each cell line did not decrease, even in response to $10 \mathrm{~Gy}$ (Fig. 1A). The clonogenic survival assay showed that U87-MG $\Delta$ EGFR cells are more resistant to radiation than are U87-MG cells, in line with the fact that it constitutively expresses EGFR (Fig. 1B).

One reason for radioresistance is that radiation transiently activates the Akt pathway, which in turn stimulates its downstream target, p70S6K, a cytoprotective molecule (24). To test whether it is the case for U87-MG and U87-MG $\triangle E$ EFF cells, we examined activation of the Akt pathway after irradiation. 10-Gy irradiation transiently increased the levels 
A
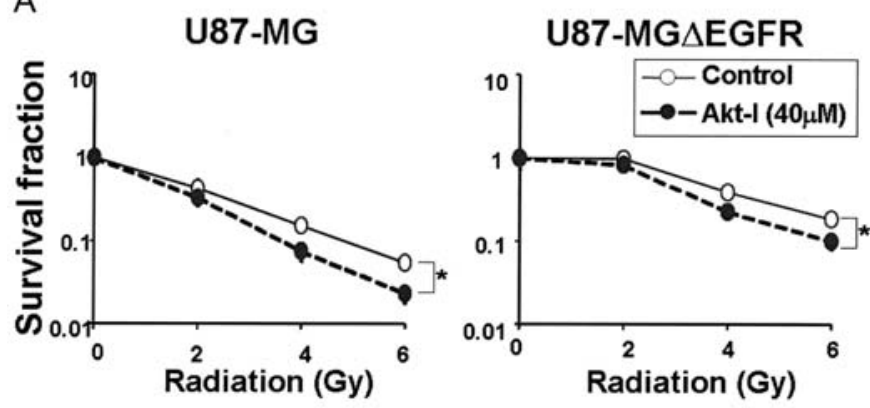

B

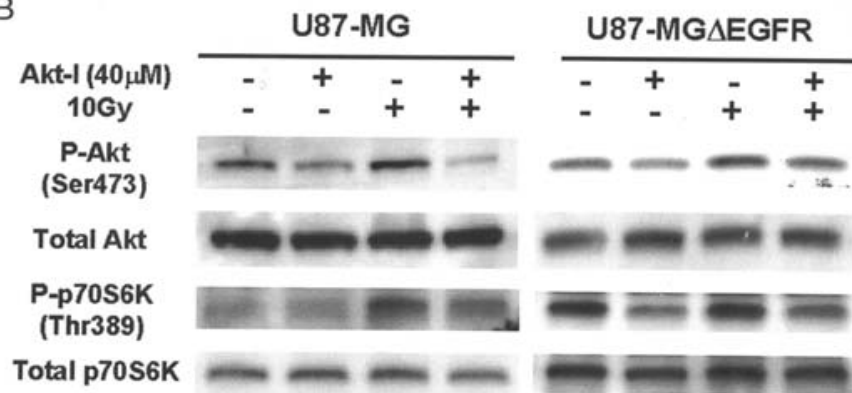

C

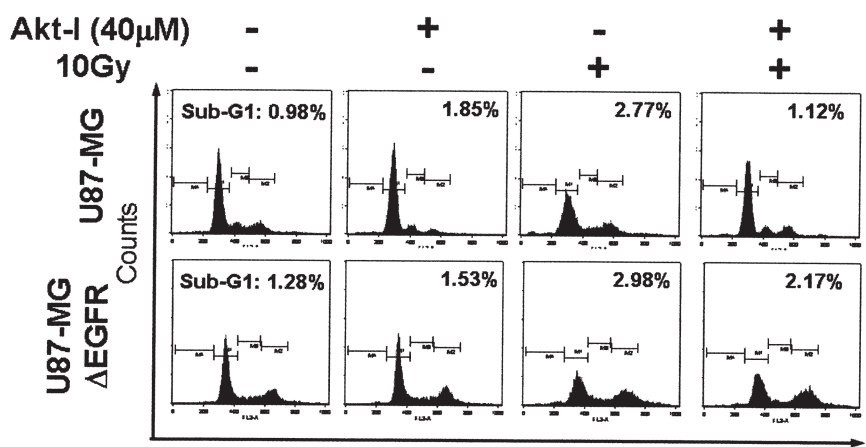

DNA content

D

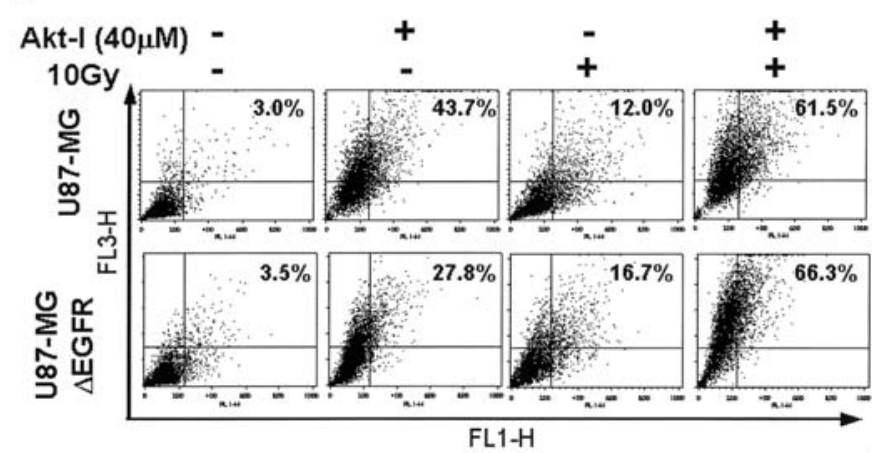

of phosphorylated Akt and phosphorylated p70S6K $\sim 1$ h later in both U87-MG and U87-MG $\Delta$ EGFR cells (Fig. 1C). These results verify that the transient activation of the Akt/p70S6K pathway in response to radiation may indeed be responsible, at least in part, for the radioresistance seen in malignant glioma cells, and that Akt inhibition is potentially a reliable means of radiosensitizing glioma cells.

The anticancer effect of Akt inhibitor in malignant glioma cells is due to induction of autophagy. Akt inhibitor was previously
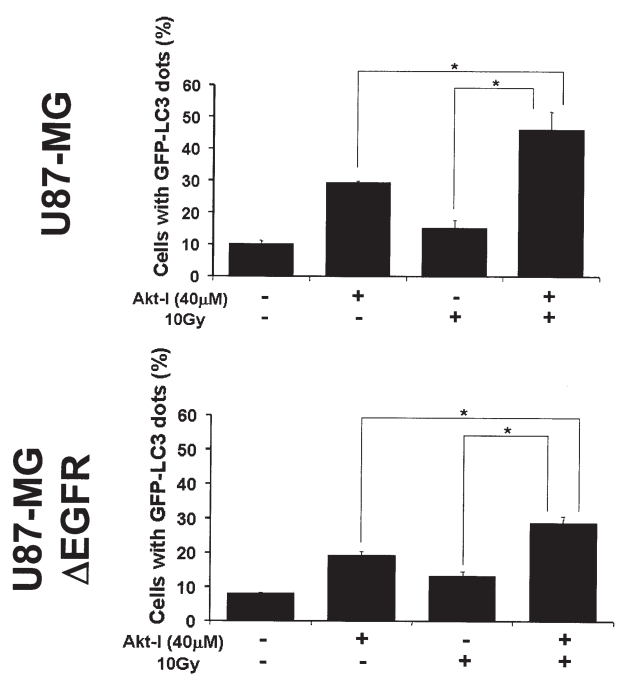

Figure 4. Akt inhibitor radiosensitizes U87-MG and U87-MG $\triangle E$ EFR cells by enhancing autophagy. A, clonogenic survival of the cells treated with combination of Akt inhibitor and radiation. Cells were treated with $40 \mu \mathrm{M}$ Akt inhibitor for $24 \mathrm{~h}$, irradiated, and plated for the assay. Values are the mean \pm SD of triplicate experiments. ${ }^{*} \mathrm{P}<0.05$. B, immunoblot analysis of the Akt/p70S6K pathway. For experiments shown in B-D, cells were treated with or without $40 \mu \mathrm{M}$ Akt inhibitor for $1 \mathrm{~h}$ and then irradiated at $10 \mathrm{~Gy}$. Cells were subjected to immunoblotting $1 \mathrm{~h}$ after irradiation. C, cell cycle analysis. Percentage of the sub-G1 population is indicated. The data shown are representative of three independent experiments. D, acridine orange staining assayed with flow cytometry. Percentage of positive-stained cells with red fluorescence is indicated. FL1-H indicates intensity of green fluorescence that is endogenous in the nucleus. FL3-H indicates intensity of red fluorescence. The data shown are representative of three independent experiments. E, quantification of the cells with GFP-LC3 dots. Values are the mean \pm SD of triplicate experiments. ${ }^{*} \mathrm{P}<0.05$.

shown to inhibit Akt activity at a low dose but not to inhibit PI3K activity, and it also inhibited cell growth in various cancer cells (21). Akt inhibitor at concentrations of $20 \mu \mathrm{M}$ and $40 \mu \mathrm{M}$ strikingly inhibited Akt activity in U87-MG and U87-MG $\triangle$ EGFR cells, respectively (Fig. 2A). It also reduced cell viability in these cells in a dose-dependent manner, with $\mathrm{IC}_{50}$ values of $\sim 25 \mu \mathrm{M}$ and $35 \mu \mathrm{M}$ for U87-MG and U87MG $\triangle E G F R$ cells, respectively (Fig. 2B).

Since Akt inhibitor was seen in a previous study not to induce apoptosis in leukemia cells except in combination with other chemotherapeutic drugs or radiation (22), we determined whether Akt inhibitor induced cell cycle arrest and apoptosis in malignant glioma cells. Our cell cycle analysis showed that Akt inhibitor induced neither cell cycle arrest nor apoptosis in either U87-MG or U87-MG $\triangle E$ EFR cells (Fig. 3A). Since the Akt/mTOR pathway also regulates autophagy, however, we then examined whether Akt inhibitor induces autophagy in these cells. Electron microscopy showed that Akt inhibitor remarkably increased the number of autophagic vacuoles containing subcellular organelles, lamellar structures, and digested residual materials in the cytoplasm of both U87-MG and U87-MG $\triangle$ EGFR cells compared with control cells (Fig. 3B).

To quantify the extent to which autophagy was induced, we determined the percentage of cells with acidic vesicular organelles using acridine orange staining. Akt inhibitor at $40 \mu \mathrm{M}$ substantially increased the percentage of cells showing 
A

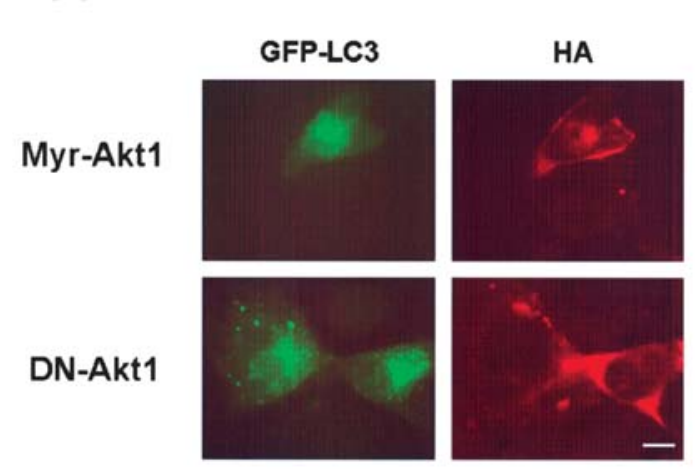

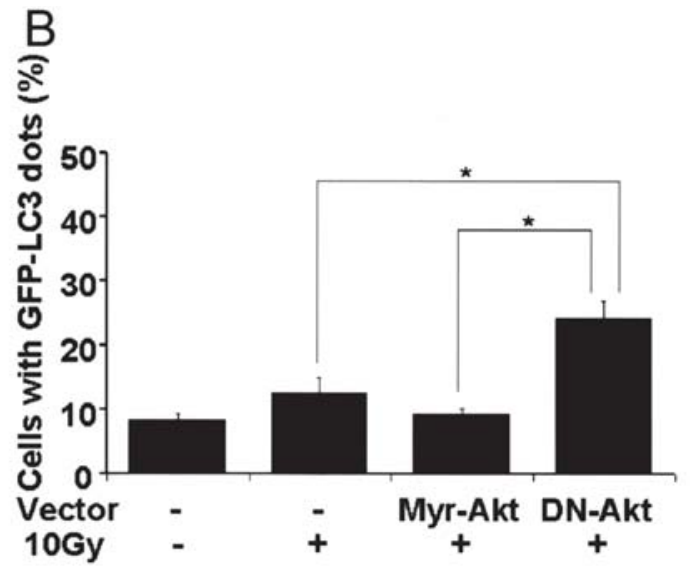

Figure 5. Specific inhibition of Akt using the DN-Akt plasmid radiosensitizes U87-MG $\triangle E G F R$ cells by enhancing autophagy. Cells were transfected with the dominant-negative Akt1 (DN-Akt1) or myristoylated Akt (Myr-Akt) plasmid. Overexpression of the exogenous proteins was confirmed with HA staining. A, photomicrographs of representative cells co-transfected with Myr-Akt1 and GFP-LC3 or DN-Akt1 and GFP-LC3. Scale bar, $10 \mu \mathrm{m}$. B, quantification of the cells with GFP-LC3 dots. Values are the mean \pm SD of triplicate experiments. ${ }^{*} \mathrm{P}<0.05$.

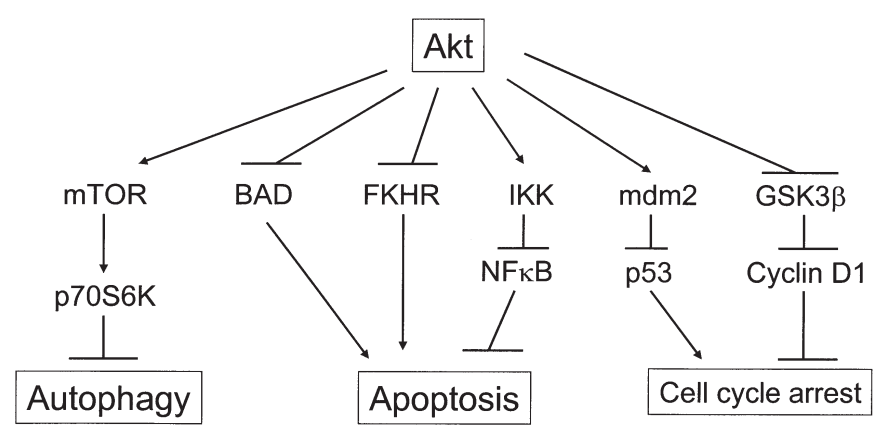

Figure 6. Signaling through the Akt pathway.

positive red staining (from 4.5 to $34.1 \%$ of U87-MG cells and from 4.3 to $24.0 \%$ of U87-MG $\triangle E$ EFR cells) (Fig. 3C).

To detect autophagosomes specifically, we next transiently transfected cells with the GFP-LC3 plasmid. LC3 is a specific marker of autophagy and it associates with the membrane of autophagosomes. During autophagy, exogenous LC3 distributes to the membrane of autophagosomes, showing characteristic GFP-LC3 dots, whereas control cells show homogeneous GFP distribution in the cytoplasm $(23,25)$. At $72 \mathrm{~h}$ after treatment with $40 \mu \mathrm{M}$ Akt inhibitor, more cells of both cell types showed GFP-LC3 dots than did the control cells (Fig. 3D). Cells with GFP-LC3 dots increased significantly from 11.9 to $38.0 \%$ of U87-MG cells and from 10.9 to $25.4 \%$ of U87-MG $\triangle E G F R$ cells ( $\mathrm{P}<0.001$ in both cell types) (Fig. 3E). These results collectively indicate that the Akt inhibitor inhibited the growth of U87-MG and U87-MG $\triangle E$ EFR cells by inducing autophagy.

Akt inhibitor radiosensitizes malignant glioma cells by enhancing autophagy. The clonogenic survival assay, done to determine whether Akt inhibitor radiosensitizes U87-MG and U87-MG $\triangle E G F R$ cells, showed that Akt inhibitor significantly radiosensitized both cell types $(\mathrm{P}<0.05)$ (Fig. 4A). In addition, the immunoblot analysis showed that Akt inhibitor effectively inhibited the radiation-induced upregulation of phosphorylated Akt and phosphorylated p70S6K in both cell types (Fig. 4B).
We also examined whether the combination of Akt inhibitor and radiation induces apoptosis or autophagy. Cell cycle analysis showed that Akt inhibitor alone, radiation alone, and the two together did not increase the sub-G1 population, indicating that Akt inhibitor did not induce apoptosis (Fig. 4C). We observed instead that 10-Gy irradiation, when used as a single treatment, induced the G2/M arrest in both cell types, which is consistent with our previous findings using other malignant glioma cell types (8). Additionally, acridine orange staining showed that Akt inhibitor further increased the percentage of irradiated cells showing positive red staining (Fig. 4D). More specifically, GFP-LC3 plasmid transfection showed that the number of U87-MG and U87-MG $\triangle E G F R$ cells with GFP-LC3 dots was significantly greater for cells treated with Akt inhibitor and radiation than for cells treated with either agent alone $(\mathrm{P}<0.05)($ Fig. 4E).

Additionally, we used the DN-Akt1 expression plasmid to verify whether specific inhibition of Akt enhances radiation-induced autophagy. This showed that significantly more cells overexpressing the DN-Akt plasmid and irradiated with 10 Gy had GFP-LC3 dots than did irradiated cells overexpressing the Myr-Akt1 and cells treated with radiation alone $(\mathrm{P}<0.05)$ (Fig. 5). These results collectively indicate that the Akt inhibitor radiosensitizes U87-MG and U87-MG $\triangle$ EGFR cells by enhancing autophagy, but not apoptosis.

\section{Discussion}

We observed that Akt inhibitor effectively inhibited Akt activity, showed cytotoxicity, and induced autophagy in both U87-MG and U87-MG $\Delta$ EGFR cells. Furthermore, Akt inhibitor radiosensitized both cell types by enhancing autophagy, but not apoptosis. We then confirmed using the DN-Akt expression plasmid that specific Akt inhibition enhanced radiation-induced autophagy. On the basis of these findings, we conclude that Akt inhibitors may represent a new strategy which can be used both as a single agent and in combination with radiation for malignant gliomas, including those with $\triangle \mathrm{EGFR}$. To the best of our knowledge, this is the 
first study to show that Akt inhibition radiosensitizes cancer cells of epithelial origin by enhancing autophagy.

Autophagy is typically observed under conditions of amino acid deprivation in mammalian cells $(11,12)$. When autophagy is induced, double-membrane structures derived from the endoplasmic reticulum or an unknown source sequester a part of the cytoplasm, often including intracellular organelles such as the mitochondrion and endoplasmic reticulum. These structures are called autophagosomes, which merge with lysosomes and then called autolysosomes. Autophagosomes and autolysosomes are collectively called autophagic vacuoles. Eventually, the inner membrane disappears and the contents inside are degraded by lysosomal hydrolases. Thus, autophagy is characterized by the prominent formation of autophagic vacuoles. Previous studies have demonstrated that autophagy occurs not only under physiologic conditions and during development, but also under some pathologic conditions such as degenerative diseases, infectious diseases, and cancer $(26,27)$. As we and others have reported, many cancer cell types undergo autophagy in response to anticancer therapies, including radiation. In the present study, we also showed that the cytotoxicity of Akt inhibitor and the extent of autophagy induced by Akt inhibitor are both dose-dependent. When used in combination with radiation, Akt inhibitor radiosensitized malignant glioma cells by enhancing autophagy. These findings suggest that autophagy is one of Akt inhibitor's anticancer effects and that enhancement of autophagy can be used as a strategy of combination therapy.

Akt, a serine-threonine kinase that regulates a wide range of target proteins, controls cell proliferation, growth, survival, and motility. Molecules downstream of Akt consist of mTOR, Forkhead (FKHR) transcription factors, glycogen synthase kinase-3 (GSK-3), mdm2, IkB kinase (IKK), and BAD $(19,20)$ (Fig. 6). In response to nutrient availability, the Akt/mTOR/ p70S6K pathway regulates autophagy and controls the growth of mammalian cells $(11,12)$. In line with this finding, Akt and p70S6K inhibit autophagy, and PTEN and rapamycin induce autophagy $(13,14,28)$. However, in the context of anticancer therapies the Akt pathway is much better known as a target to induce cell cycle arrest and apoptosis than a target to induce autophagy. Multiple mechanisms through Akt to inhibit cell cycle arrest and apoptosis have been identified. For example, the phosphorylation of GSK-3ß by Akt inhibits the phosphorylation of cyclin D1, thereby blocking the degradation of cyclin D1 by the proteasome. Akt inactivates pro-apoptotic factors including BAD, procaspase-9, and FKHR transcription factors. Akt also inhibits apoptosis by modulating mdm 2 and IKK: The phosphorylation of mdm2 counteracts the tumor suppressor gene product $\mathrm{p} 53$; the phosphorylation of IKK activates the nuclear translocation of nuclear factor of $\kappa \mathrm{B}(\mathrm{NF}-\kappa \mathrm{B})$, resulting in inhibition of apoptosis.

Though Akt has various functions, Akt inhibitors do not always inhibit all of these functions. In the present study we showed that Akt inhibitor induced autophagy, but not either apoptosis or cell cycle arrest in malignant glioma cells. A previous study using the same Akt inhibitor also showed that Akt inhibitor itself did not induce apoptosis in leukemia cells that are resistant to various treatment, though it was found to decrease antiapoptotic proteins Mcl-1 and FLIP, and decreased phosphorylated BAD (22). However, Akt inhibitor did enhance apoptosis in the same cell type when used in combination with conventional anticancer therapies. The effect of Akt inhibitors on the cell cycle was not documented in that study. Nonetheless, a recent study showed that perifosine, another Akt inhibitor, inhibited Cdk2 activity and induced the G1 and G2 cell cycle arrest in glial progenitor cells overexpressing the platelet-derived growth factor (PDGF) (29). The reasons why Akt inhibitors induce apoptosis in some cell types and autophagy in other cell types are not clear. It is possible that this depends on the cells' susceptibility to apoptosis, as suggested by the finding that perifosine induced apoptosis in control glial progenitor cells but not in glial progenitor cells overexpressing PDGF (29). Since the overexpression of PDGF makes cells less susceptible to apoptosis, it is likely that blocking the Akt pathway induces autophagy in cancer cells of epithelial origin, including malignant glioma cells, which are very resistant to apoptosis. That is, in these resistant cancer cells, apoptotic pathways are suppressed or disrupted, whereas autophagic pathways are still functioning. In line with this, a recent study showed that rapamycin radiosensitized breast cancer cells by enhancing autophagy (30).

In summary, we observed that Akt inhibitor successfully inhibited Akt activity and induced autophagy, but not apoptosis, in U87-MG and U87-MG $\Delta$ EGFR cells. Thus, enhancing autophagy is a new means of gauging the effects of various anticancer therapies. Further, Akt inhibitor radiosensitized both cell types by enhancing autophagy. Akt inhibitors may thus represent a new strategy for radiosensitizing cancer cells that are resistant to apoptosis in response to anticancer therapies. New Akt inhibitors have been developed in the hope of inhibiting Akt directly and strongly, and producing less adverse side-effects compared with inhibitors of other targets of the PI3K/Akt pathway. These Akt inhibitors include Akt antibodies, phosphatidyl inositol analogues such as the one used in this study and perifosine, ATP-competitive inhibitors, pseudosubstrate inhibitors, and Akt inhibitors identified from screening a huge collection of compounds $(28,31)$. Based on the results of the present study, these new drugs are expected to be effective not only as single agents, but also as agents used in combination with conventional anticancer therapies including radiotherapy.

\section{Acknowledgements}

We thank Dr Noboru Mizushima for his kind gift of the GFPLC3 plasmid. We also thank Ms. E. Faith Hollingsworth for her technical help, Ms. Betty Notzon for editing the manuscript, and the High Resolution Electron Microscopy Facility for assistance with the electron microscopy. This study was supported by Institutional Research Grant from The University of Texas M.D. Anderson Cancer Center (Y. Kondo), USPHS Grants CA088936 and CA108558 (S. Kondo), Institutional Cancer Core Grant (CA16672), and a generous donation from the Anthony D. Bullock III Foundation (S. Kondo and Y. Kondo).

\section{References}

1. Kondo Y, Kanzawa T, Sawaya R and Kondo S: The role of autophagy in cancer development and response to therapy. Nat Rev Cancer 5: 726-734, 2005. 
2. Gozuacik D and Kimchi A: Autophagy as a cell death and tumor suppressor mechanism. Oncogene 23: 2891-2906, 2004.

3. Ogier-Denis E and Codogno P: Autophagy: a barrier or an adaptive response to cancer. Biochim Biophys Acta 1603: 113-128, 2003.

4. Bursch W, Hochegger K, Torok L, Marian B, Ellinger A and Hermann RS: Autophagic and apoptotic types of programmed cell death exhibit different fates of cytoskeletal filaments. J Cell Sci 113: 1189-1198, 2000

5. Kanzawa T, Germano IM, Komata T, Ito H, Kondo $\mathrm{Y}$ and Kondo S: Role of autophagy in temozolomide-induced cytotoxicity for malignant glioma cells. Cell Death Differ 11: 448-457, 2004.

6. Paglin S, Hollister T, Delohery T, et al: A novel response of cancer cells to radiation involves autophagy and formation of acidic vesicles. Cancer Res 61: 439-444, 2001.

7. Yao KC, Komata T, Kondo Y, Kanzawa T, Kondo S and Germano IM: Molecular response of human glioblastoma multiforme cells to ionizing radiation: cell cycle arrest, modulation of the expression of cyclin-dependent kinase inhibitors, and autophagy. J Neurosurg 98: 378-384, 2003.

8. Ito H, Daido S, Kanzawa T, Kondo S and Kondo Y: Radiationinduced autophagy is associated with LC3 and its inhibition sensitizes malignant glioma cells. Int J Oncol 26: 1401-1410, 2005.

9. Daido S, Yamamoto A, Fujiwara K, Sawaya R, Kondo S and Kondo Y: Inhibition of the DNA-dependent protein kinase catalytic subunit radiosensitizes malignant glioma cells by inducing autophagy. Cancer Res 65: 4368-4375, 2005.

10. Clarke PG: Developmental cell death: morphological diversity and multiple mechanisms. Anat Embryol 181: 195-213, 1990.

11. Klionsky DJ and Emr SD: Autophagy as a regulated pathway of cellular degradation. Science 290: 1717-1721, 2000.

12. Meijer AJ and Codogno P: Regulation and role of autophagy in mammalian cells. Int J Biochem Cell Biol 36: 2445-2462, 2004.

13. Takeuchi H, Kondo Y, Fujiwara K, et al: Synergistic augmentation of rapamycin-induced autophagy in malignant glioma cells by phosphatidylinositol 3-kinase/protein kinase B inhibitors. Cancer Res 65: 3336-3346, 2005.

14. Arico S, Petiot A, Bauvy C, et al: The tumor suppressor PTEN positively regulates macroautophagy by inhibiting the phosphatidylinositol 3-kinase/protein kinase B pathway. J Biol Chem 276: 35243-35246, 2001

15. Sugawa N, Ekstrand AJ, James CD and Collins VP: Identical splicing of aberrant epidermal growth factor receptor transcripts from amplified rearranged genes in human glioblastomas. Proc Natl Acad Sci USA 87: 8602-8606, 1990

16. Huang HS, Nagane M, Klingbeil CK, et al: The enhanced tumorigenic activity of a mutant epidermal growth factor receptor common in human cancers is mediated by threshold levels of constitutive tyrosine phosphorylation and unattenuated signaling. J Biol Chem 272: 2927-2935, 1997.
17. Li B, Yuan M, Kim IA, Chang CM, Bernhard EJ and Shu HK Mutant epidermal growth factor receptor displays increased signaling through the phosphatidylinositol-3 kinase/AKT pathway and promotes radioresistance in cells of astrocytic origin. Oncogene 23: 4594-4602, 2004.

18. Lammering G, Hewit TH, Valerie K, et al: EGFRvIII-mediated radioresistance through a strong cytoprotective response. Oncogene 22: 5545-5553, 2003.

19. West KA, Castillo SS and Dennis PA: Activation of the PI3K/ Akt pathway and chemotherapeutic resistance. Drug Resist Updates 5: 234-248, 2002.

20. Hennessy BT, Smith DL, Ram PT, Lu Y and Mills GB Exploiting the PI3K/AKT pathway for cancer drug discovery. Nat Rev Drug Discov 4: 988-1004, 2005.

21. Hu Y, Qiao L, Wang S, et al: 3-(Hydroxymethyl)-bearing phosphatidylinositol ether lipid analogues and carbonate surrogates block PI3-K, Akt, and cancer cell growth. J Med Chem 43: 3045-3051, 2000

22. Martelli AM, Tazzari PL, Tabellini G, et al: A new selective AKT pharmacological inhibitor reduces resistance to chemotherapeutic drugs, TRAIL, all-trans-retinoic acid, and ionizing radiation of human leukemia cells. Leukemia 17: 1794-1805, 2003

23. Kabeya Y, Mizushima N, Ueno T, et al: LC3, a mammalian homologue of yeast Apg8p, is localized in autophagosome membranes after processing. EMBO J 19: 5720-5728, 2000.

24. Contessa JN, Hampton J, Lammering G, et al: Ionizing radiation activates Erb-B receptor-dependent Akt and p70 S6 kinase signaling in carcinoma cells. Oncogene 21: 4032-4041, 2002.

25. Kirisako T, Baba M, Ishihara N, et al: Formation process of autophagosome is traced with Apg8/Aut7p in yeast. J Cell Biol 147: 435-446, 1999.

26. Shintani T and Klionsky DJ: Autophagy in health and disease: a double-edged sword. Science 306: 990-995, 2004.

27. Cuervo AM: Autophagy: in sickness and in health. Trends Cell Biol 14: 70-77, 2004.

28. Blommaart EF, Luiken JJ, Blommaart PJ, van Woerkom GM and Meijer AJ: Phosphorylation of ribosomal protein S6 is inhibitory for autophagy in isolated rat hepatocytes. J Biol Chem 270: 2320-2326, 1995.

29. Momota H, Nerio E and Holland EC: Perifosine inhibits multiple signaling pathways in glial progenitors and cooperates with temozolomide to arrest cell proliferation in gliomas in vivo. Cancer Res 65: 7429-7435, 2005.

30. Paglin S, Lee NY, Nakar C, et al: Rapamycin-sensitive pathway regulates mitochondrial membrane potential, autophagy, and survival in irradiated MCF-7 cells. Cancer Res 65: 11061-11070, 2005.

31. Cheng JQ, Lindsley CW, Cheng GZ, Yang H and Nicosia SV: The Akt/PKB pathway: molecular target for cancer drug discovery. Oncogene 24: 7482-7492, 2005. 